\title{
Microsecond Structural Rheology
}

\author{
Felix Lehmkühler, ${ }^{*, \dagger, \ddagger}$ Ingo Steinke, ${ }^{\dagger, \ddagger}$ Martin A. Schroer,,,$+ \uparrow$ Birgit Fischer, ${ }^{\dagger, t, \S}$ \\ Michael Sprung, ${ }^{\dagger}$ and Gerhard Grübel ${ }^{\dagger, \ddagger}$ \\ $\dagger$ †eutsches Elektronen-Synchrotron DESY, Notkestr. 85, 22607 Hamburg, Germany \\ $\ddagger$ The Hamburg Centre for Ultrafast Imaging (CUI), Luruper Chaussee 149, 22761 \\ Hamburg, Germany \\ Icurrent address: European Molecular Biology Laboratory EMBL c/o DESY, Notkestr. 85, \\ 22607 Hamburg, Germany \\ $\S c u r r e n t$ address: Institut für Physikalische Chemie, Universität Hamburg, Grindelallee \\ 117, 20146 Hamburg, Germany \\ E-mail: felix.lehmkuehler@desy.de
}




\begin{abstract}
The relationship between the local structure of complex liquids and their response to shear is generally not well understood. This concerns in particular the formation of particle strings in flow direction or hydroclusters, both important for the understanding of shear thinning and thickening phenomena. Here, we present results of a microfocus X-ray scattering experiment on spherical silica colloids in a liquid jet at high shear rates. Along and across the jet we observe direction-dependent modifications of the structure factor of the suspension suggesting the formation of differently ordered clusters in stream and compression lines. With increasing distance from the orifice the structure relaxes to the unsheared case with a typical relaxation 10 times larger as the time scale of Brownian motion. These results provide the first experimental flow characterization of a complex fluid at high shear rates detecting cluster formation and relaxation with micrometer and microsecond resolution.
\end{abstract}

\title{
Graphical TOC Entry
}

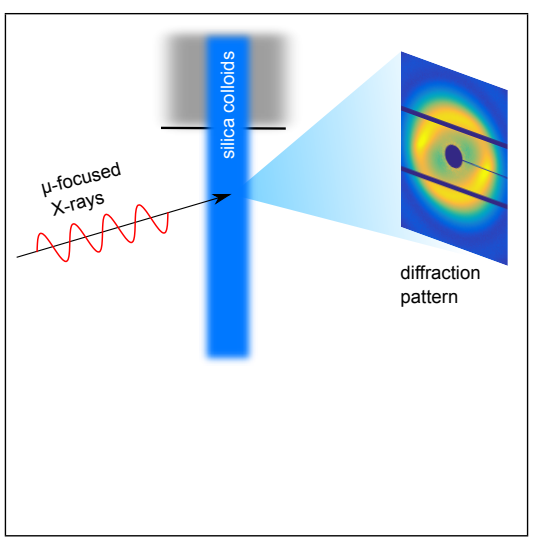


The response of concentrated colloidal dispersions to shear has risen scientific and technological interest in the last decades. ${ }^{1,2}$ Common features of flowing dense dispersions are shear thinning and thickening, ${ }^{3-9}$ i.e. the reduction or increase of the suspension's viscosity with shear rate. In those systems, Brownian motion is dominated by shear forces. Hydrodynamic forces cannot be neglected leading to a coupling of particles which may drive shear thickening and the formation of so-called hydrocluster. ${ }^{2,5}$ Though these phenomena have been studied in numerous simulation studies (see e.g. the recent work by Marenne et al. ${ }^{10}$ ), experiments studying structure formation under rheological conditions and the formation of clusters or studying the connection between structure and shearing are missing. For structural studies of these phenomena, two conditions have to be fulfilled: (1) An experimental probe that is sensitive to local order has to be applied and (2) the experiment has to be performed at high shear rates where such clusters typically form. In a pioneering study by Cheng et al. ${ }^{5}$ the authors performed fast confocal microscopy to measure real space arrangements of particles. This technique provides access to the real space local structure of small parts of the sample, but it is still limited to rather slow systems to obtain structural snapshots with single exposures. In contrast, scattering methods allow to access sample structures down to molecular length scales.Examples are classical light scattering work on dense hard sphere systems reporting ordering phenomena under shear. ${ }^{3,11}$ In such experiments, the probing beam, either visible light, ${ }^{12,13}$ neutrons ${ }^{14,15}$ or X-rays, ${ }^{16,17}$ is scattered from the sample in standard rheological geometries. The spatial resolution, e.g. for scanning across the sample away from the moving plate, is given by the beam size which is typically limited to about $100 \mu \mathrm{m}$ in small-angle scattering experiments using X-rays (SAXS) or neutrons (SANS), respectively, providing ensemble averaged structural information over different structural realisations due to shear. This can be improved by using state-of-the-art instruments providing focused X-rays, allowing for beam sizes around and below few $\mu \mathrm{m}$. Such beam sizes enable to use microfluidic devices, e.g. Poiseuille flow geometries, to perform structural rheology experiments, ${ }^{18-20}$ allowing access to high shear rates in the regime of $\dot{\gamma} \approx 10^{5}-10^{6} \mathrm{~s}^{-1} \cdot{ }^{21}$ 
These shear rates correspond to the regime where structural modulations due to shear are expected. ${ }^{2}$ By this approach, structural response of soft matter systems on shear can be studied with high spatial resolution.

A particular representation of such a microfluidic device is a liquid microjet. Here, the most simple jet version is the so-called Rayleigh jet where a liquid is pressed through a thin tube producing a steady-streaming liquid jet. Its size is defined by the dimension of the tube. After a certain distance $L$ depending on different geometrical parameters and properties of the liquid, these jets break up into droplets. Limits of the break-up length of Rayleigh jets can be defined via the Reynolds and Weber numbers. ${ }^{22-24}$ With the increasing use of liquid microjets as sample carrier in current X-ray scattering experiments, in particular structural studies on biological macromolecules at FEL sources ${ }^{25-27}$ or orientation of non-isotropic particles, ${ }^{28,29}$ properties of liquid jets have generated renewed scientific interest. Structural rheology properties of jets are especially of importance in order to understand alignment or ordering of macromolecules under shear.

In this work, we investigate the structural rheology of a dispersion of spherical colloidal silica particles in water in a Rayleigh jet using a microfocused X-ray beam. Taking scattering patterns at different positions across and along the jet we measure the impact of shear on the static structure factor $S(q)$ of the particles originating from the flow through a microtube. We observed direction-dependent modulations of particle-particle distances as proposed by simulations, ${ }^{30,31}$ suggesting the formation of ordered strings of particles in flow direction and compression lines under shear. In addition, we measure the timescale of relaxation back to an undisturbed structure after shear cessation.

The investigated jet is characterized by an average speed of $v_{\text {jet }}=3.5 \mathrm{~m} / \mathrm{s}$, a shear rate of $\dot{\gamma}=2.8 \times 10^{5} \mathrm{~s}^{-1}$ and thus a Péclet number of $\mathrm{Pe} \approx 8$ (see Methods for details). SAXS patterns were taken at different distances $h$ between $100 \mu \mathrm{m}$ and $3000 \mu \mathrm{m}$ downstream of the tube exit. At these locations diffraction patterns were recorded across the jet in steps of $\Delta d=1 \mu \mathrm{m}$ with a beamsize of $6 \mu \mathrm{m} \times 3 \mu \mathrm{m}$. A scheme of the jet properties is given in Fig. 1 . 


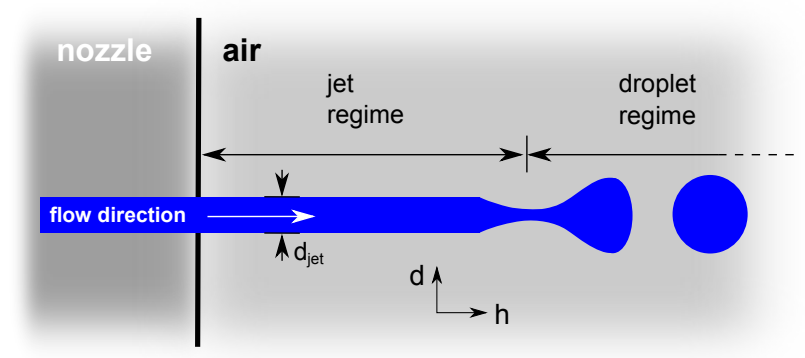

Figure 1: Scheme of the liquid jet. The liquid is pressed through the nozzle resulting in a jet with a thickness of $d_{\text {jet }} \approx 100 \mu \mathrm{m}$. At the break-up length $L$ the jet breaks into a train of droplets.

The coordinate system is defined by directions $h$ (along the jet) and $d$ (across the jet). The origin is set to the jet center at the tube exit. The definition of coordinates $h$ and $d$ as well as the angle $\omega$ relative to the flow direction is given in Fig. 2 (a). Exemplary scattering patterns are shown in Fig. 2 at $h=100 \mu \mathrm{m}$, i.e. $100 \mu \mathrm{m}$ downstream the tube exit, and across the jet at $d=-30 \mu \mathrm{m}(\mathrm{b}), d=0 \mu \mathrm{m}(\mathrm{c})$, and $d=30 \mu \mathrm{m}(\mathrm{d})$, respectively. First, we estimate the jet thickness from the total scattering intensity probed by the detector. Neglecting the anyway weak absorption of X-rays by the jet, the total scattering intensity can be assumed to increase linearly with the probed thickness of the jet. Assuming a cylindrical jet shape, the probed thickness can be described by the chord length of a circle with diameter $d_{\text {jet }}$. In this way the fit to the data yields a jet diameter of $d_{\text {jet }}=(102 \pm 8) \mu \mathrm{m}$ at $h=100 \mu \mathrm{m}$ after deconvolution with the beam shape, in good agreement to the tube diameter of 100 $\mu \mathrm{m}$, see Fig. 2 (e). Taking a closer look on the diffraction patterns, the pattern at $d=0$ $\mu \mathrm{m}$ is isotropic and resembles the scattering pattern of non-flowing particles in capillaries (not shown), while towards the edges of the jet the patterns become anisotropic with respect to shape and intensity. Furthermore, the patterns show a mirror symmetry with respect to the $d=0 \mu \mathrm{m}$ as shown here for $d= \pm 30 \mu \mathrm{m}$ in Fig. $2(\mathrm{~b}),(\mathrm{d})$. These observations are representative for all distances $h$ studied.

For spherical particles the scattering intensity as function of wave vector transfer $\mathbf{q}$ is given by $I(\mathbf{q}) \propto P(\mathbf{q}) S(\mathbf{q})$ with the particle form factor $P(\mathbf{q})$, which is well known for 

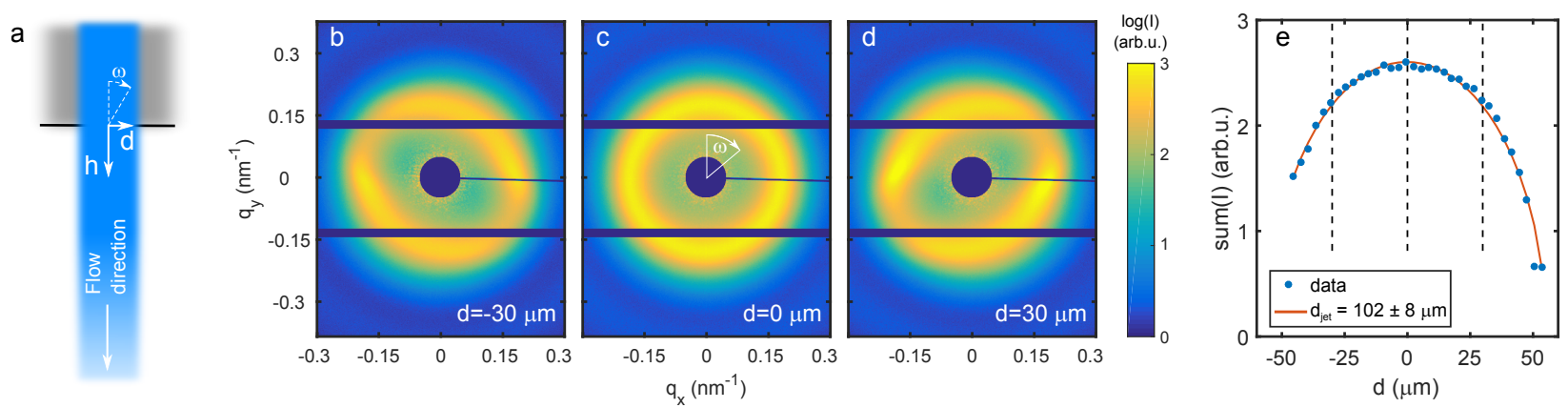

Figure 2: Diffraction patterns taken at $h=100 \mu \mathrm{m}$ across the jet. (a) Definition of coordinates $d$ and $h$ and angle $\omega$ used for the scattering signal relative to the flow direction of the jet. (b) $d=-30 \mu \mathrm{m}$. (c) $d=0 \mu \mathrm{m}$ (d) $d=30 \mu \mathrm{m}$. (e) Total recorded scattering intensity across the jet together with fit of the jet diameter. The dashed lines mark the positions $d$ discussed in (b-d).

spheres $^{32}$ and only depends on the modulus $q=|\mathbf{q}|$, and the static structure factor $S(\mathbf{q})$. Thus, the asymmetry of the diffraction patterns suggests an anisotropic ordering of the silica particles resulting in an asymmetric $S(\mathbf{q})$ with respect to the azimuthal angle $\omega$. Therefore, we extract structure factor maps from the diffraction patterns. For this purpose the diffraction patterns were binned in azimuthal segments of $\Delta \omega=2^{\circ}$ width and integrated to obtain $I(\mathbf{q})=I(q, \omega)$. Afterwards, the segments $I(q, \omega)$ were divided by $P(q)$ obtained from a form factor fit of these spheres in a diluted suspension. Resulting $S(q, \omega)$-maps are shown in Fig. 3 (a) and (b). The asymmetry becomes obvious for $d=30 \mu \mathrm{m}$ in Fig. 3 (a), with strong, non-isotropic maxima at approximately $\omega_{1}=\pi / 4$ and $\omega_{2}=\pi / 2$. In contrast, at the center of the jet $(d=0 \mu \mathrm{m}) S(\mathbf{q})$ is isotropic as observed already from the diffraction patterns. These observations are underlined by cuts of the $S(q)$-maps along $\omega_{1}, \omega_{2}$ and for comparison $\omega_{3}=3 \pi / 4$ shown in Fig. 3 (c) and (d), respectively. In the following we use the $q$-position $q_{0}$ and the amplitude $S_{0}$ of the maximum of the structure factors $S(q, \omega)$ to describe observations quantitatively. Only small differences can be observed for $d=0 \mu \mathrm{m}$. Changes of the position $q_{0}$ and the amplitude $S_{0}$ at $d=30 \mu \mathrm{m}-$ and correspondingly at $d=-30 \mu \mathrm{m}-$ suggest variations of direction-dependent ordering and volume fraction in the jet. The absolute maximum value of $S_{0}$ can be found at approximately $\omega_{2}$ perpendicular to the jet flow with $q_{0}=0.20 \mathrm{~nm}^{-1}$. This indicates that the order is best defined in this di- 
rection, suggesting the formation of co-flowing strings of particles with well-defined distance across the jet direction. Towards the center and at the edges of the jet this is less defined.

The variation of ordering becomes even more obvious for the modulation of $q_{0}$ as function of $\omega$. This is shown in Fig. 4 (a) for the three $d$ values at $h=100 \mu \mathrm{m}$ discussed above. Here, for $d=0 \mu \mathrm{m}$ the static structure factor for a non-flowing dispersion, with peak position $q^{*}$ and amplitude $S^{*}$ is well reproduced. We find this peak at $q^{*}=0.2 \mathrm{~nm}^{-1}$. The structure factor for $d=0 \mu \mathrm{m}$ can be approximately described within the rescaled mean spherical approximation (RMSA) model of charged spheres. ${ }^{33,34}$ Therein, the structure factor is described as a function of the particles' volume fraction, particles' charge, solvent permittivity and salt concentration in the solvent. Across the jet, the RMSA model fails to describe the structure factors within the same set of parameters. Nevertheless, from the variation of $q_{0}$, strong modulations of the local structure can be concluded. Furthermore, the $S(q)$ curves have a mirror symmetry with respect to $\omega=\pi$, and $q_{0}$ varies between approximately $0.185 \mathrm{~nm}^{-1}$ and $0.23 \mathrm{~nm}^{-1}$.
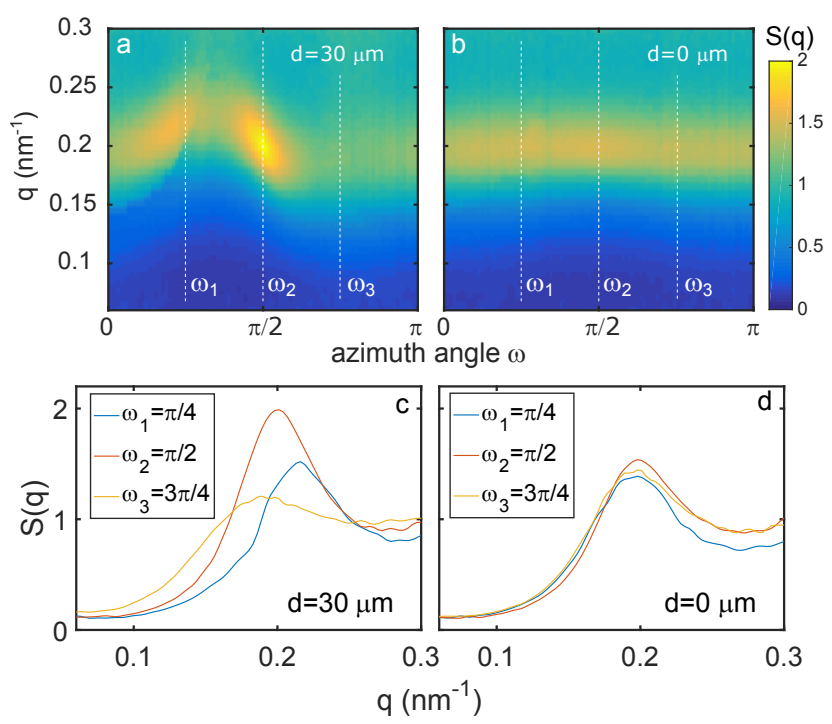

Figure 3: Structure factor maps at $h=100 \mu \mathrm{m}$. Due to Friedel's symmetry $(I(-\mathbf{q})=I(\mathbf{q}))$, structure factors are averaged for the intervals $[0, \pi]$ and $[2 \pi, \pi]$, respectively. (a) $d=30$ $\mu \mathrm{m}$. (b) $d=0 \mu \mathrm{m}$. (c) and (d) are cuts along $\omega_{1}=\pi / 4, \omega_{2}=\pi / 2$, and $\omega_{3}=3 \pi / 4$ as indicated by the dashed lines in (a) and (b).

In order to quantify the structural modification across and along the jet, we introduce 
different order parameters. First, in Fig. 4 (b) variances $\operatorname{var}\left(q_{0}\right)=\left.\operatorname{var}\left(q_{0}(d)\right)\right|_{\omega}$ (top) and $\operatorname{var}\left(S_{0}\right)=\left.\operatorname{var}\left(S_{0}(d)\right)\right|_{\omega}$ (bottom) are shown at different values of $h$ and $d$. The observations from Figs. 4 (b) are well reproduced, i.e. weak modifications in the jet center accompanied by stronger modulations towards the edges, while the variation decreases close to the edge. With increasing distance to the nozzle exit the peaks become weaker and they move towards the center of the jet, suggesting reduced appearance of direction-depended order in the jet. In the droplet regime measured around $h=3000 \mu \mathrm{m}$, no modulation across the droplets can be observed. In Fig. 4 (c) the maximum and minimum values of $q_{0}$ are shown as function of d. The data are normalized to the non-sheared sample, i.e. to $q^{*}=0.2 \mathrm{~nm}^{-1}$. The highest variation is found around $d= \pm 30 \mu \mathrm{m}$, similar to the observations in Fig. 4 (b). This decreases monotoneously towards the jet center $(d=0 \mu \mathrm{m})$ and the edges of the jet. Due to scattering from the jet edges leading to streaks in the scattering patterns, ${ }^{35}$ only positions $d$ have been analyzed that were not affected by such parasitic scattering.
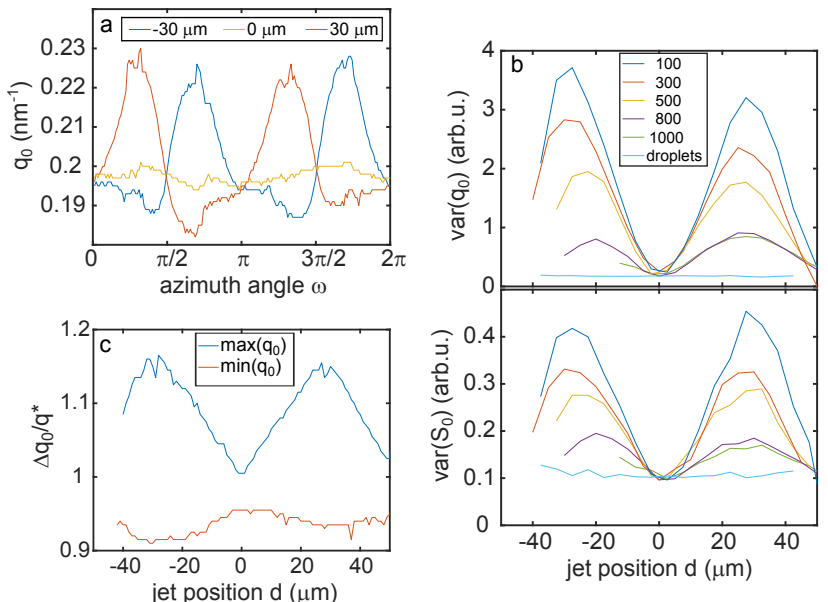

Figure 4: Variations along and across the jet. (a) $q_{0}$ for three horizontal positions across the jet at $h=100 \mu \mathrm{m}$. (b) Variance of $q_{0}$ (top) and $S_{0}$ (bottom) with respect to $\omega$ across the jet at different distances to the nozzle exit as indicated in the legend. (c) Normalized shift of $\max _{\omega}\left(q_{0}\right)$ and $\min _{\omega}\left(q_{0}\right)$ with respect to $q^{*}$ as a function of $d$ for $h=100 \mu \mathrm{m}$.

The direction-dependent change of $q_{0}$ and thus of the volume fraction $\phi$ is quantified in Fig. 5. Therein, in panel (a) the results of high resolution scans, i.e. steps of $\Delta d=1 \mu \mathrm{m}$ are shown for $h=100 \mu \mathrm{m}$ and $h=1000 \mu \mathrm{m}$. A rougher overview is given in panel (b) 
where a stepsize of $\Delta d=5 \mu \mathrm{m}$ was used, resembling the beam size in horizontal direction of $6 \mu \mathrm{m}$. At each point across the jet a line or arrow starts whose magnitude is given by the order parameter $\xi(d, h)=\max _{\omega}\left(q_{0}(d, h, \omega)\right)-\min _{\omega}\left(q_{0}(d, h, \omega)\right)$ discussed in Fig. 4 (b). The direction is defined by the azimuthal location of the maximum of $q_{0}$, i.e. around $\pi / 4$ for $h=100 \mu \mathrm{m}$ and $d=30 \mu \mathrm{m}$, cf. Fig. 4 (a). Thus, the arrows provide the direction and amplitude of the decrease of particle-particle distance due to shear from the tube wall. Interestingly, the effects are highest at around $d= \pm 30 \mu \mathrm{m}= \pm 0.6 r_{\text {jet }}$ and less pronounced in the jet center and close to the edges. For all distances and positions, the minimum of the particle-particle distance is oriented about a tilt angle of approximately $\pi / 4$ towards the jet center for all distances. This observation is in line with results from simulations ${ }^{2,10,30}$ where a compression of particles along axes of $\pm \pi / 4$ has been reported above a Péclet number of $\mathrm{Pe} \approx 1$. The amplitude of $\xi$ decreases with $h$ as a consequence of structural relaxation to a non-sheared fluid downstream of the tube. Taking into account the average jet speed of $v=3.5 \mathrm{~m} / \mathrm{s}$ (see Method section) the $h$-axis can be translated to a time axis, see right axes in Fig. 5 (a) and (b). In this way, $\xi$ can be studied as function of the liquid's travelling time $t$ where $t=0 \mathrm{~s}$ defines the location of the tube exit. In Fig. 5 (c) $\xi$ is shown as function $t / t_{b}$, with the time of Brownian motion $t_{b}=R^{2} / D$ that defines the time scale of diffusion. $D$ denotes the diffusion constant of free silica particles with radius $R$ in water (see Experimental Methods). The drop of $\xi$ is modelled by an exponential decay $\xi(t)=A \exp (-\Gamma t)$. The fit yields a decay time of $\tau=1 / \Gamma=(10 \pm 1) t_{b}=(288 \pm 29) \mu \mathrm{s}$, that is a measure of relaxation of the sheared-induced order.Thus, we obtained a microsecond time scale of shear cessation ${ }^{36,37}$ which can hardly be accessed by other techniques. Remarkably, this is significant slower than observed in recent simulations of dense hard spheres $(\phi=0.4)$ where the time scale of cessation was found to be $\tau_{\mathrm{HS}}<t_{b}{ }^{10}$ Therefore, an extension of simulations to charge stabilized systems under shear which is object of our study will provide valuable information on structure formation and shear cessation of colloidal systems.

In conclusion, we reported the first $\mu \mathrm{m}$ resolution structural rheology study at high shear 

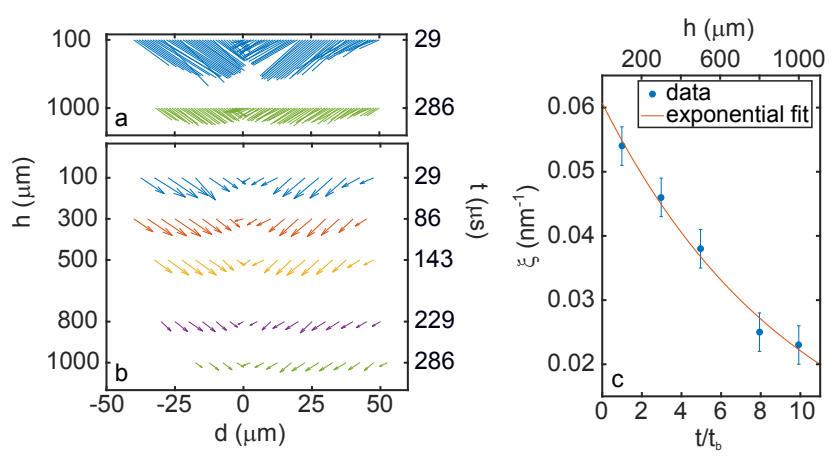

Figure 5: Orientation and amplitude of compression lines along and across the jet. (a) High resolution with steps of $1 \mu \mathrm{m}$. (b) Steps of $5 \mu \mathrm{m}$. (c) Order parameter $\xi$ as a function of $t$ normalized to the Brownian time $t_{b}$.

rates $(\mathrm{Pe} \approx 8)$ using a liquid jet. We found the formation of direction-dependent order in a prototypical soft matter system of silica particles dispersed in water. In general, it is most prominent at $d= \pm 0.6 r_{\text {jet }}$ and decreases towards the center and close to the edges of the jet. Two main features of direction dependent order are observed: (1) The order expressed by $S_{0}$ is best defined perpendicular to the jet flow, suggesting the formation of co-flowing strings of particles and (2) the particles are compressed along $\pm \frac{\pi}{4}$ relative to the direction of flow. Furthermore, since the sample is only sheared inside the tube, the jet offers to study structure relaxation back to the unsheared liquid by measuring the sample structure along the jet. For our sample we observe a relaxation time of $\tau=10 t_{b}$. Access to these properties in conventional structural rheology experiments is limited by the minimum exposure and counting time. In contrast, the use of a liquid jet transfers the time axis to a spatial axis whose resolution is given by the beam size. In our case, we achieved a time resolution of $\Delta t \approx 1 \mu \mathrm{s}$, unprecendented in any experimental rheology approach so far.

\section{Experimental Methods}

The experiment was performed at beamline P10 at PETRA III (DESY, Hamburg, Germany) in SAXS geometry at an X-ray energy of $7 \mathrm{keV}$. The sample-detector distance was set to 5 m, using a Dectris Pilatus 300k detector. The beam was focused by compound refractive 
lenses to $6 \mu \mathrm{m} \times 3 \mu \mathrm{m}$ (horizontal $\times$ vertical). As Rayleigh nozzle a micro tube (Polymicro GmbH, inner diameter $d_{\mathrm{i}}=100 \mu \mathrm{m}$, outer diameter $\left.d_{\mathrm{o}}=375 \mu \mathrm{m}\right)$ was used inside the sample chamber described elsewhere ${ }^{29}$ defining a jet thickness of $d_{\text {jet }}=100 \mu \mathrm{m}$. On each jet location an exposure time of $t_{e}>10 \mathrm{~s}$ was used. As sample we used a commercially available dispersion of silica particles (Sigma-Aldrich, Ludox TMA 420859) at a volume fraction of about $\phi=19$ vol\%. The unsheard samples were characterized by SAXS and modeled within $\mathrm{RMSA}^{33,34}$ yielding a particle radius of $R=12.9 \mathrm{~nm}$ with a size polydispersity of $\Delta R / R=0.12$, a particle charge of $z=(45 \pm 2) e_{0}$ with the electron charge $e_{0}$, and an ionic strength of charge screening material due to reminder of co-solvents in the solution of $c=(10 \pm 4) \mu \mathrm{M}$. A flow rate of approximately $Q \approx 27.8 \mu \mathrm{l} / \mathrm{h}$ was set by nitrogen pressure used to press the liquid through the tube. Thus, the average flow velocity can be estimated to $v_{\text {jet }} \approx 3.5 \mathrm{~m} / \mathrm{s}$, leading to a shear rate for Newtonian fluids in a pipe of $\dot{\gamma}=\frac{8 v_{\text {jet }}}{d_{\text {jet }}}=2.8 \times 10^{5} \mathrm{~s}^{-1}$ and a Péclet number of $\mathrm{Pe}=\frac{6 \pi \eta R^{3} \dot{\gamma}}{k_{\mathrm{B}} T}=\frac{\dot{\gamma} R^{2}}{D} \approx 8$, with the diffusion constant $D=\frac{k_{\mathrm{B}} T}{6 \pi \eta R}$, and Boltzman's constant $k_{\mathrm{B}}$, temperature $T$, and viscosity $\eta$.

\section{Acknowledgement}

The authors thank Michael Walther for technical support. Parts of this research were carried out at the light source PETRA III at DESY, a member of the Helmholtz Association (HGF). The Hamburg Centre for Ultrafast Imaging (CUI) and the Graduate School 1355 of the Deutsche Forschungsgemeinschaft (DFG) are acknowledged for financial support. 


\section{References}

(1) Macosko, C. Rheology. Principles, Measurement, and Applications; Wiley-VCH: New York, USA, 1994.

(2) Wagner, N.; Brady, J. Shear Thickening in Colloidal Dispersions. Phys. Today 2009, 62, 27-32.

(3) Hoffman, R. Discontinuous and Dilatant Viscosity Behavior in Concentrated Suspensions. I. Observation of a Flow Instability. Trans. Soc. Rheol. 1972, 16, 155-173.

(4) Brown, E.; Forman, N.; Orellana, C.; Zhang, H.; Maynor, B.; Betts, D.; DeSimone, J.; Jaeger, H. Generality of Shear Thickening in Dense Suspensions. Nat. Mater. 2010, 9, $220-224$.

(5) Cheng, X.; McCoy, J.; Israelachvili, J.; Cohen, I. Imaging the Microscopic Structure of Shear Thinning and Thickening Colloidal Suspensions. Science 2011, 333, 1276-1279.

(6) Takeda, M.; Kusano, T.; Matsunaga, T.; Endo, H.; Shibayama, M.; Shikata, T. RheoSANS Studies on Shear-Thickening/Thinning in Aqueous Rodlike Micellar Solutions. Langmuir 2011, 27, 1731-1738.

(7) Seto, R.; Mari, R.; Morris, J.; Denn, M. Discontinuous Shear Thickening of Frictional Hard-Sphere Suspensions. Phys. Rev. Lett. 2013, 111, 218301.

(8) Brown, E.; Jaeger, H. M. Shear Thickening in Concentrated Suspensions: Phenomenology, Mechanisms and Relations to Jamming. Rep. Prog. Phys. 2014, 7r, 046602.

(9) Wyart, M.; Cates, M. Discontinuous Shear Thickening without Inertia in Dense NonBrownian Suspensions. Phys. Rev. Lett. 2014, 112, 098302.

(10) Marenne, S.; Morris, J. F.; Foss, D. R.; Brady, J. F. Unsteady Shear Flow of Colloidal Hard-Sphere Suspensions by Dynamic Simulation. J. Rheol. 2017, 61, 477-501. 
(11) Ackerson, B. J. Shear Induced Order and Shear Processing of Model Hard Sphere Suspensions. J. Rheol. 1990, 34, 553-590.

(12) Clark, N. A.; Ackerson, B. J. Observation of the Coupling of Concentration Fluctuations to Steady-State Shear Flow. Phys. Rev. Lett. 1980, 44, 1005-1008.

(13) Dhont, J. K. G.; Lettinga, M. P.; Dogic, Z.; Lenstra, T. A. J.; Wang, H.; Rathgeber, S.; Carletto, P.; Willner, L.; Frielinghaus, H.; Lindner, P. Shear-Banding and Microstructure of Colloids in Shear Flow. Faraday Discuss. 2003, 123, 157-172.

(14) Yearley, E. J.; Sasa, L. A.; Welch, C. F.; Taylor, M. A.; Kupcho, K. M.; Gilbertson, R. D.; Hjelm, R. P. The Couette Configuration of the Los Alamos Neutron Science Center Neutron Rheometer for the Investigation of Polymers in the Bulk via SmallAngle Neutron Scattering. Rev. Sci. Instrum. 2010, 81, 045109.

(15) Eberle, A. P.; Porcar, L. Flow-SANS and Rheo-SANS Applied to Soft Matter. Curr. Opin. Colloid Interface Sci. 2012, 17, 33-43.

(16) Panine, P.; Gradzielski, M.; Narayanan, T. Combined Rheometry and Small-Angle X-ray Scattering. Rev. Sci. Instrum. 2003, 74, 2451-2455.

(17) Denisov, D.; Dang, M.; Struth, B.; Wegdam, G.; Schall, P. Resolving Structural Modifications of Colloidal Glasses by Combining X-ray Scattering and Rheology. Sci. Rep. 2013, 3, 1631.

(18) Nordstrom, K. N.; Verneuil, E.; Arratia, P. E.; Basu, A.; Zhang, Z.; Yodh, A. G.; Gollub, J. P.; Durian, D. J. Microfluidic Rheology of Soft Colloids above and below Jamming. Phys. Rev. Lett. 2010, 105, 175701.

(19) Trebbin, M.; Steinhauser, D.; Perlich, J.; Buffet, A.; Roth, S. V.; Zimmermann, W.; Thiele, J.; Förster, S. Anisotropic Particles Align Perpendicular to the Flow Direction in Narrow Microchannels. Proc. Natl. Acad. Sci. 2013, 110, 6706-6711. 
(20) Wieland, D. C. F.; Zander, T.; Garamus, V. M.; Krywka, C.; Dedinaite, A.; Claesson, P.; Willumeit-Römer, R. Complex Solutions under Shear and Pressure: a Rheometer Setup for X-ray Scattering Experiments. J. Synchrotron Rad. 2017, 24, 646-652.

(21) Wieland, D. C. F.; Garamus, V. M.; Zander, T.; Krywka, C.; Wang, M.; Dedinaite, A.; Claesson, P. M.; Willumeit-Römer, R. Studying Solutions at High Shear Rates: a Dedicated Microfluidics Setup. J. Synchrotron Rad. 2016, 23, 480-486.

(22) Sterling, A. M.; Sleicher, C. A. The Instability of Capillary Jets. J. Fluid Mech. 1975, $68,477-495$.

(23) Leroux, S.; Dumouchel, C.; Ledoux, M. The Stability Curve of Newtonian Jets. Atomization Spray. 1996, 6, 623-647.

(24) Lin, S. Breakup of Liquid Sheets and Jets; Cambridge University Press: Cambridge, UK, 2003.

(25) Chapman, H. N.; Fromme, P.; Barty, A.; White, T. A.; Kirian, R. A.; Aquila, A.; Hunter, M. S.; Schulz, J.; DePonte, D. P.; Weierstall, U. et al. Femtosecond X-ray Protein Nanocrystallography. Nature 2011, 470, 73.

(26) Boutet, S.; Lomb, L.; Williams, G. J.; Barends, T. R. M.; Aquila, A.; Doak, R. B.; Weierstall, U.; DePonte, D. P.; Steinbrener, J.; Shoeman, R. L. et al. High-Resolution Protein Structure Determination by Serial Femtosecond Crystallography. Science 2012, $337,362$.

(27) Oberthuer, D.; Knoska, J.; Wiedorn, M. O.; Beyerlein, K. R.; Bushnell, D. A.; Kovaleva, E. G.; Heymann, M.; Gumprecht, L.; Kirian, R. A.; Barty, A. et al. Double-Flow Focused Liquid Injector for Efficient Serial Femtosecond Crystallography. Sci. Rep. 2017, r, 44628 . 
(28) Priebe, M.; Kalbfleisch, S.; Tolkiehn, M.; Köster, S.; Abel, B.; Davies, R. J.; Salditt, T. Orientation of Biomolecular Assemblies in a Microfluidic Jet. New J. Phys. 2010, 12, 043056.

(29) Steinke, I.; Walther, M.; Lehmkühler, F.; Wochner, P.; Valerio, J.; Mager, R.; Schroer, M. A.; Lee, S.; Roseker, W.; Jain, A. et al. A Liquid Jet Setup for X-ray Scattering Experiments on Complex Liquids at Free-Electron Laser Sources. Rev. Sci. Instrum. 2016, 87, 063905.

(30) Foss, D.; Brady, J. Structure, Diffusion and Rheology of Brownian Suspensions by Stokesian Dynamics Simulation. J. Fluid Mech. 2000, 407, 167-200.

(31) Kulkarni, S. D.; Morris, J. F. Ordering Transition and Structural Evolution under Shear in Brownian Suspensions. J. Rheol. 2009, 53, 417-439.

(32) de Jeu, W. H. Basic X-Ray Scattering for Soft Matter; Oxford University Press: Oxford, 2016.

(33) Hansen, J.-P.; Hayter, J. B. A Rescaled MSA Structure Factor for Dilute Charged Colloidal Dispersions. Mol. Phys. 1982, 46, 651-656.

(34) Westermeier, F.; Fischer, B.; Roseker, W.; Grübel, G.; Nägele, G.; Heinen, M. Structure and Short-Time Dynamics in Concentrated Suspensions of Charged Colloids. J. Chem. Phys. 2012, 137, 114504.

(35) Marmiroli, B.; Cacho-Nerin, F.; Sartori, B.; Pérez, J.; Amenitsch, H. Thorough SmallAngle X-ray Scattering Analysis of the Instability of Liquid Micro-Jets in Air. J. Synchrotron Rad. 2014, 21, 193-202.

(36) Kielhorn, L.; Colby, R. H.; Han, C. C. Relaxation Behavior of Polymer Blends after the Cessation of Shear. Macromolecules 2000, 33, 2486-2496. 
(37) Ianni, F.; Lasne, D.; Sarcia, R.; Hébraud, P. Relaxation of Jammed Colloidal Suspensions after Shear Cessation. Phys. Rev. E 2006, 74, 011401. 